\title{
Electron Microscopic Investigation of Anterior Lens Capsule and Epithelium in Patients with Diabetes Mellitus
}

\author{
Yasemin Ozdamar Erol ${ }^{*}$, Candan Ozogul², Ufuk Elgin ${ }^{3}$, Fatma Helvacioglu², Orhan Zilelioglu ${ }^{3}$ \\ ${ }^{1}$ Department of Retinal Diseases and Vitreoretinal Surgery, Ulucanlar Eye Educationand Research Hospital, \\ Ankara, Turkey \\ ${ }^{2}$ Department of Histology and Embryology, Faculty of Medicine, Gazi University, Ankara, Turkey \\ ${ }^{3}$ Ulucanlar Eye Educationand Research Hospital, Ankara, Turkey \\ Email: *yasemin_oz@yahoo.com
}

How to cite this paper: Erol, Y.O., Ozogul, C., Elgin, U., Helvacioglu, F. and Zilelioglu, O. (2018) Electron Microscopic Investigation of Anterior Lens Capsule and Epithelium in Patients with Diabetes Mellitus. International Journal of Clinical Medicine, 9, 778-786. https://doi.org/10.4236/ijcm.2018.910064

Received: June 8, 2018

Accepted: October 26, 2018

Published: October 29, 2018

Copyright (c) 2018 by authors and Scientific Research Publishing Inc. This work is licensed under the Creative Commons Attribution International License (CC BY 4.0).

http://creativecommons.org/licenses/by/4.0/

\begin{abstract}
Purpose: To evaluate the histopathologic alterations of organelles in the epithelium of the anterior lens capsule. Methods: The interventional study included 26 eyes; of which 11 had non-proliferative diabetic retinopathy (diabetic group) and cataract, and 15 had age-related cataract (control group). We investigated the anterior lens capsule in patients with diabetes mellitus by using electron microscopy and to compare it between diabetic eyes and healthy eyes. Anterior capsule samples were obtained by circular continuous capsulorhexis during phacoemulsification procedures. All the samples were fixed and conventionally processed for electron microscopy analysis. Results: Demographic characteristics of the diabetic group and the control group were similar $(\mathrm{p}>0.05)$. In the diabetic group, electron dense cells with an apoptotic appearance were seen and these cells had an apoptotic nucleus and prominent mitochondrial crystalysis. In addition, there was dilatation of the endoplasmic reticulum cistern. In the control group, lens epithelial cells and all their elements had a normal pattern. Neither cells with an apoptotic appearance nor mitochondrial crystalysis was seen, but there was dilatation of the endoplasmic reticulum cistern. Conclusions: Diabetes mellitus can engender structural abnormalities of organelles in the epithelium of the anterior lens capsule including mitochondrial crystalysis, dilatation of the endoplasmic reticulum cistern and apoptotic dense nucleus. It can be suggested that diabetes mellitus affects organelles of anterior lens epithelium in eyes with cataracts, while it causes non-proliferative changes in the retina.
\end{abstract}

\section{Keywords}

Anterior Lens Epithelium, Cataract, Diabetes Mellitus, Endoplasmic 
Reticulum, Mitochondria

\section{Introduction}

Diabetes mellitus (DM) is the most significant cause of blindness in the world. Diabetes-related reactions are responsible for structural and functional changes in the eyes, and these changes are suspected of contributing to development of long-term diabetic complications like cataracts, glaucoma and retinopathy if DM is not kept under control well [1] [2].

Cataracts are one of the complications of DM starting the earliest. How excess glucose initiates cataract development in patients with diabetes mellitus has not been explained yet. Many of the common factors including sorbitol formation associated with aldose reductase activity, oxidative stress, protein glycation and formation of advanced glycation end products (AGE) have been implicated in diabetic cataract development [3] [4] [5]. The structure of the normal lens is almost perfectly transparent, which is regulated by physical and chemical processes. Disruption of these processes in the lens capsule and the epithelium can result in lens opacification and cataract formation [6]. However, histopathologic alterations of organelles in the epithelium of the anterior lens capsule with diabetic eyes have not been studied previously. Therefore, we investigated transmission electron microscopic findings of the epithelium of the anterior lens capsule in patients with DM and compared them with those from age matched controls.

\section{Patients and Methods}

This clinical prospective interventional study was performed at University of HealthSciences-Ulucanlar EyeEducationandResearchHospital. The study protocol was approved by the ethics committee of the hospital and the study was carried out in accordance with the Declaration of Helsinki. Written informed consent was obtained from all the patients included in the study.

The study comprised a total of 26 eyes with cataracts, of which 11 belonged to patients with Type II DM consecutively presenting to hospital (Diabetic Group = DG) and 15 belonged to nondiabetic patients with age related cataract (Control Group = CG). The diabetic patients were outpatients at Retina Department of Hospital and they had non-proliferative diabetic retinopathy. In both groups, anterior lens capsules were obtained during elective cataract surgery. Exclusion criteria included trauma, uveitis, glaucoma, history of previous intraocular surgery and history of long-term systemic or ocular therapy and systemic diseases. Cataracts were in nuclear or cortical forms in all the eyes and the subjects with mature or white cataract morphology were excluded from the study.

Glycosylated hemoglobin (HbA1c) levels were determined. Diabetic patients with $\mathrm{HbA1c}>10$ were not included in the study and DG only included type II-diabetic patients. All the patients were examined by an internal physician be- 
fore surgery and non-diabetic patients with normal basal glycaemia were included in the study. None of the participants in the control group had ocular diseases except for senile cataracts.

All the cases underwent a comprehensive ophthalmic examination including best corrected visual acuity using the Snellen chart (20 feet), intraocular pressure measurement, slit lamp biomicroscopy and fundoscopy preoperatively. Results of blood tests including complete blood count, liver and renal function tests, fasting glucose levels and $\mathrm{HbA1c}$ levels were evaluated by an experienced internal specialist and the patients eligible for phacoemulsification surgery were determined.

All the cases underwent uncomplicated phacoemulsification surgery under local anesthesia. All the operations were performed by the same experienced surgeon (YOE). The anterior chamber was entered with a $2.8 \mathrm{~mm}$ keratome at the limbus. An ophthalmic viscosurgical device was injected into the anterior chamber. An anterior capsule flap was created with a cystotome and a circle of the central capsule with a diameter of $5-5.5 \mathrm{~mm}$ was carefully removed by continuous curvilinear capsulorhexis with forceps. The anterior capsule was fixed immediately. The lens capsule was not stained in all eyes.

The samples of the anterior lens capsule were kept in $2.5 \%$ glutaraldehyde for 24 hours for primary fixation. Then, these samples were washed with Sorenson's Phosphate Buffer solution ( $\mathrm{pH}: 7.4$ ) and post-fixed in $1 \%$ osmium tetroxide. After post-fixation, they were washed with the same buffer and dehydrated in increasing concentrations of alcohol series. Following dehydration, the tissues were washed with propylene oxide and embedded in epoxy resin embedding media. The semi-thin and ultrathin sections of the obtained tissue blocks were cut with an ultramicrotome (LKB Nova, Sweden). These semi-thin sections, which were 2 micrometers in thickness, were stained with methylene blue and examined under a light microscope (Nikon, Japan). Following this procedure, the tissue blocks were exposed to trimming and their ultrathin sections, which were about 60 nanometers in thickness, were taken by the same ultramicrotome. These ultra-thin sections were stained with uranyl acetate and lead citrate and examined under Jeol JEM 1200 EX (Japan) transmission electron microscope. The electron micrographs of the specimens were taken by the same microscope. Anterior lens capsule wrinkles were not observed during the preparation of the tissues.

Mann-Whitney $\mathrm{U}$ test and One-sample Chi-square test were used to compare the demographics characteristics. $\mathrm{P}<0.05$ was considered statistically significant. Statistical analyses were performed with the Statistical Package for the Social Sciences for Windows (SPSS, version 10.0, Inc., Chicago, IL, USA).

\section{Results}

The study included 26 eyes of 26 patients, of whom 11 formed the diabetic group (DG) and the remaining 15 formed the control group (CG). DG consisted of 5 males (45.5\%) and 6 females (54.5\%) and the mean age of this group was $67.18 \pm$ 
6.38 years. CG included 7 males (46.7\%) and 8 females (53.3\%) with a mean age of $65.38 \pm 7.25$ years. HbA1c levels were between 5.9 and 10 in DG. There were no significant differences in age and gender between CG and DG ( $p>0.05)$.

Electron microscopic examination of the anterior lens capsules revealed significant ultrastructural changes in DG when compared to CG. In DG, electron dense cells with an apoptotic appearance were seen and these cells had an apoptotic nucleus and prominent mitochondrial crystalysis. Also, there was dilatation in the endoplasmic reticulum cisterns (Figure 1 and Figure 2). In CG, lens epithelial cells and all their elements had a normal pattern. Neither cells with apoptotic appearance nor mitochondrial crystalysis was observed, but there was dilatation in the endoplasmic reticulum cistern (Figure 3 and Figure 4).

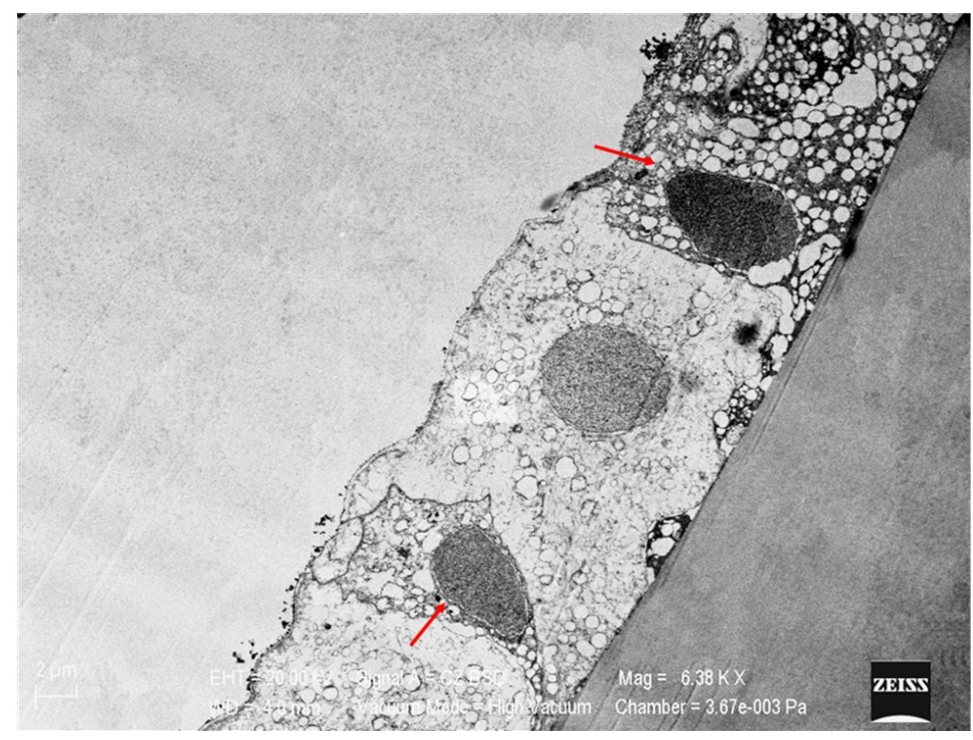

Figure 1. The appearance of apoptotic cells (red arrow) in DG.

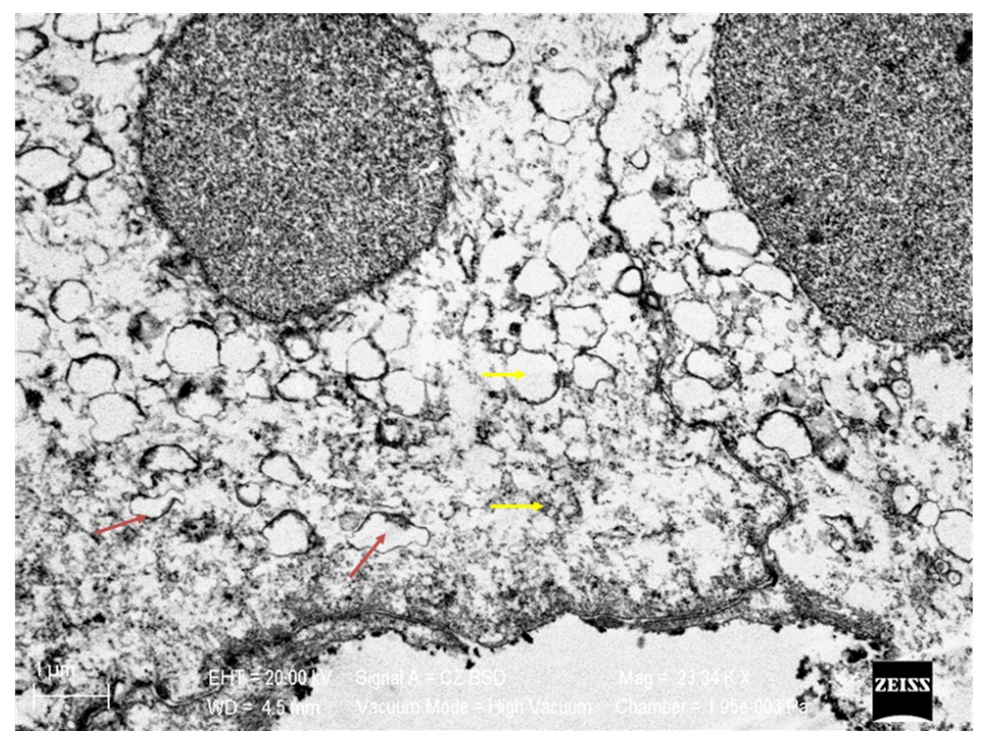

Figure 2. The dilatation of endoplasmic reticulum cistern (red arrow) and mitochondrial crystalysis (yellow arrow) in DG. 


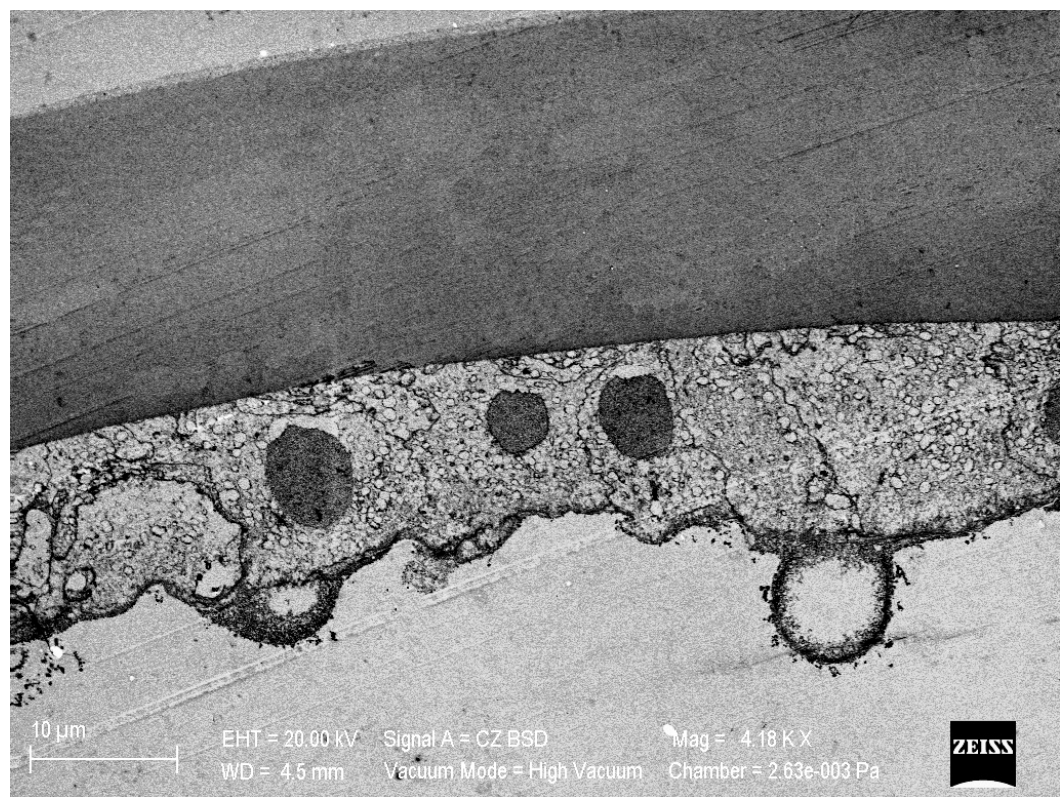

Figure 3. The normal epithelial cell in CG.

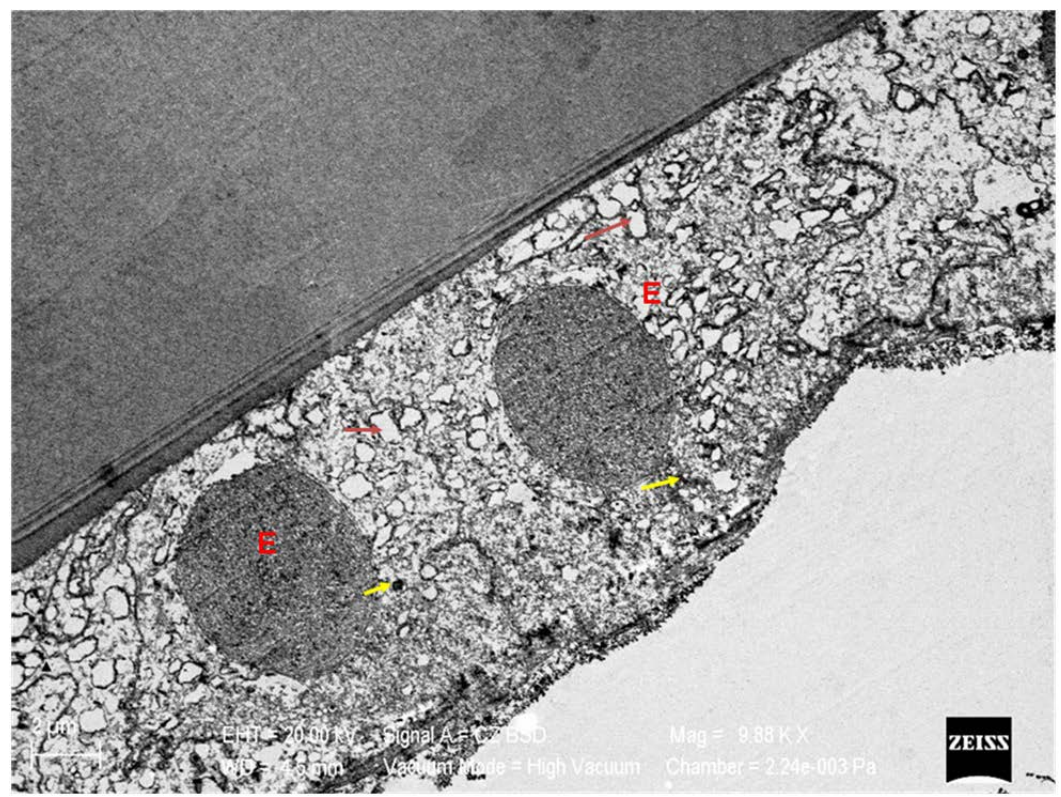

Figure 4. The normal appearance of epithelial cell and mitochondria (yellow arrow) and the dilatation of endoplasmic reticulum cistern (red arrow) in CG.

\section{Discussion}

Histologically, the structure of the lens comprises the lens capsule, the lens epithelium and the lens fiber [7] [8] [9] [10]. A modified transparent basement membrane secreted by the lens epithelial cells, the lens capsule is the thickest basement membrane having a specific structure and functions in the human body. The lens epithelium exhibits a characteristic epithelial morphology. Anterior lens epithelial cells are flattened cuboidal hexagonal in shape, are tightly packed in a single layer with very little intercellular space and contain a round 
nucleus with a few apically distributed organelles, i.e. ribosomes, polysomes, smooth and rough endoplasmic reticulum (ER), and Golgi bodies. These cells have small mitochondria with irregular cristae. Each mitochondrion is surrounded by a smooth outer membrane and a folded inner membrane. The cristae, the folds of the inner membrane, considerably increase the surface area of the inner membrane and the surface area for ATP synthesis and reactions related to electron transport, Krebs citric acid cycle, and oxidative phosphorylation. Histologically, ER is made up of interconnected tubules and vesicles, the lumens of which are called the cistern, and it is the main site of protein synthesis [11] [12].

The lens capsule and the lens epithelium act as a regulating barrier between the aqueous humor and the lens fibers. Also, they allow for passive exchanging of metabolic substrates, expelling of wastes and selectively filtering molecules based on size and charge. They play a crucial role in preservation of lens transparency. Any factor which disturbs the transport processes, morphology or biochemistry of the lens capsule and the epithelium would lead to cataract formation [6] [13].

The present study was directed towards investigating transmission electron microscopic findings of organelles in the anterior lens capsule epithelium with DM and comparing them with those from age matched controls. All the diabetic anterior capsules in this study showed significant ultrastructural changes. To the best our knowledge, this is the first study to evaluate histopathological alterations in organelles of the anterior lens capsule epithelium of diabetic cataracts.

Cataracts are considered a major cause of visual impairment in patients with DM [14]. However, the exact pathogenesis of diabetic cataract development is completely unknown. Several factors including oxidative stress and generation of reactive oxygen species (ROS), aldose reductase and sorbitol pathway hyperactivity, and protein glycation and formation of AGEs have been held responsible for the development of diabetic cataracts in recent studies [3] [4] [5].

Until today, aldose reductase and sorbitol pathway hyperactivity have been reported in many studies to be associated with diabetic cataracts and it has been shown that increased intracellular sorbitol leads to localized osmotic stress. It is also known that intracellular accumulation of sorbitol and AGEs in the human lens leads to osmotic stress and hydropic degeneration of lens fibers. Increased osmotic stress initiates an apoptotic process in epithelial cells contributing to the cataract formation [3] [4] [5] [15]. Furthermore, aldose reductase and sorbitol pathway hyperactivity have been proposed to initiate oxidative stress and generation of ROS [16]. Moreover, it has been reported in many studies that chronic hyperglycemia may cause oxidative stress and formation of ROS [15]. Hyperglycemia related to mitochondrial dysfunction is the basic source of formation of oxidative stress specimens [17]. Additionally, stress of ER resulting from osmotic stress is another source of oxidative specimens [15] [18]. It can be estimated that mitochondrial dysfunction and ER stress in lens epithelial cell accelerate 
lens opacification [14]. In addition, formation of AGEs through non-enzymatic glucose-protein reactions has been implicated in excessive glucose levels in DM. Normal accumulation of AGEs has also been observed in normal aging process [19].

Adults who have type 2 diabetes display cortical and/or posterior subcapsular opacities, which are often accompanied by nuclear sclerosis and which resemble typical senile cataracts of nondiabetics. Despite their morphological similarities, lenses with cataracts in diabetics have lower epithelial cell densities than those in healthy individuals [20] [21] [22]. The capsule and the epithelium of eyes with cataracts have morphological features, typical of aging cells such as altered hexagonal cellular arrays, and changes in the endoplasmic reticulum, the Golgi apparatus and the mitochondria [23].

In the present study, epithelial cells in abnormal lens with an apoptotic nucleus with prominent mitochondrial crystalysis were observed in the anterior capsules of the diabetic patients. It can be suggested that these ultrastructural changes may be caused by intracellular accumulation of sorbitol and AGEs and osmotic stress and associated with over expression of intrinsic mitochondrial apoptotic pathways in the lens capsules of these patients.

It has been noted in the literature that osmotic stress creates stress in the ER in diabetic patients [24] [25]. The stress in the ER initiates a series of protective signal transduction pathways from ER to the cytoplasm and nucleus, known collectively as the unfolded-protein response (UPR) [12]. The amount of unfolded protein can be monitored in the ER lumen. When it is higher than a certain threshold, ER sensors activate a signal transduction pathway. The responses activated by this pathway are referred to the UPR. Several cellular insults impair protein folding and lead to deposition of unfolded protein in the ER lumen. If there is not an adequate UPR adaptive response to repair the protein folding defect, apoptotic death of the cells starts. Furthermore, apoptosis is activated through cell death pathways which include multiple caspases and the release of produced reactive oxygen species and these outcomes may also lead to a UPR response [12] [24] [25]. It can be suggested that accumulation of UPR might have led to dilatation of ER cisterns in anterior lens capsules of the patients with DM in the current study. Additionally, the formation of reactive oxygen species and age-related AGE products might have caused retention of UPR and dilatation of ER cisterna in the control group.

The limitations of this study are that the study sample was small and only included non-proliferative diabetic eyes with type II DM. For this reason, the findings cannot be generalized to all diabetic patients.

In conclusion, DM can lead to some significant changes including apoptotic dense nucleus, prominent mitochondrial crystalysis and dilated endoplasmic reticulum in the anterior lens epithelium of eyes with non-proliferative stage. It can be suggested that diabetes mellitus may affect organelles of anterior lens epithelium in eyes with cataracts, while it causes non-proliferative changes in the 
retina. It can be expected that understanding of the complex mechanisms would lead to discovery and development of innovative therapeutic intervention strategies for diabetic patients.

\section{Acknowledgements}

This research received no specific grant from any funding agency in the public, commercial, or not-for-profit sectors. The authors report no conflicts of interest and have no proprietary interest in any of the materials mentioned in this article. All authors have contributed significantly, and that all authors are in agreement with the content of the manuscript.

\section{Conflicts of Interest}

The authors declare no conflicts of interest regarding the publication of this paper.

\section{References}

[1] Brian, G. and Taylor, H. (2001) Cataract Blindness-Challenges for the $21^{\text {st }}$ Century. Bulletin of the World Health Organization, 79, 249-256.

[2] Sayin, N., Kara, N. and Pekel, G. (2015) Ocular Complications of Diabetes Mellitus. World Journal of Diabetes, 6, 92-108. https://doi.org/10.4239/wjd.v6.i1.92

[3] Tang, W.H., Martin, K.A. and Hwa, J. (2012) Aldose Reductase, Oxidative Stress, and Diabetic Mellitus. Frontiers in Pharmacology, 3, 87. https://doi.org/10.3389/fphar.2012.00087

[4] Snow, A., Shieh, B., Chang, K.C., Pal, A., Lenhart, P., Ammar, D., Ruzycki, P., Palla, S., Reddy, G.B. and Petrash, J.M. (2015) Aldose Reductase Expression as a Risk Factor for Cataract. Chemico-Biological Interactions, 234, 247-253. https://doi.org/10.1016/j.cbi.2014.12.017

[5] Hashim, Z. and Zarina, S. (2012) Osmotic Stress Induced Oxidative Damage: Possible Mechanism of Cataract Formation in Diabetes. Journal of Diabetes and Its Complications, 26, 275-279. https://doi.org/10.1016/j.jdiacomp.2012.04.005

[6] Danysh, B.P. and Duncan, M.K. (2009) Thelens Capsule. Experimental Eye Research, 88, 151-164. https://doi.org/10.1016/j.exer.2008.08.002

[7] Danysh, B.P., Patel, T.P., Czymmek, K.J., Edwards, D.A., Wang, L., Pande, J. and Duncan, M.K. (2010) Characterizing Molecular Diffusion in the Lens Capsule. Matrix Biology, 29, 228-236. https://doi.org/10.1016/j.matbio.2009.12.004

[8] Cohen, A.I. (1965) The Electron Microscopy of the Normal Human Lens. Investigative Ophthalmology \& Visual Science, 4, 433-446.

[9] Truscott, R.J. (2005) Age-Related Nuclear Cataract-Oxidation Is the Key. Experimental Eye Research, 80, 709-725. https://doi.org/10.1016/j.exer.2004.12.007

[10] Bhat, S.P. (2001) The Ocular Lens Epithelium. Bioscience Reports, 21, 537-563. https://doi.org/10.1023/A:1017952128502

[11] Kuszak, J.R., Clark, J.I., Cooper, K.E. and Rae, J.L. (2008)Biology of the Lens: Lens Transparency as a Function of Embryology, Anatomy, and Physiology. In: Azar, D.T. and Rosen, E.S., Eds., Albert \& Jakobiec's Principles and Practice of Ophthalmology, 3rd Edition, Elsevier, Philadelphia, 1291-1339.

[12] Ovalle, W.K. and Nahirney, P.C. (2013) The Cell. In: Netter's Essential Histology, 
2nd Edition, Saunders/Elsevier, Philadelphia, 1, 1-28.

[13] Andjelic, S., Drašlar, K., Hvala, A., Lopic, N., Strancar, J. and Hawlina, M. (2015) Anterior Lens Epithelial Cells Attachment to the Basal Lamina. Acta Ophthalmologica, 94, e183-188. https://doi.org/10.1111/aos.12902

[14] Pollreisz, A. and Schmidt-Erfurth, U. (2010) Diabetic Cataract-Pathogenesis, Epidemiology and Treatment. Journal of Ophthalmology, 2010, 8. https://doi.org/10.1155/2010/608751

[15] Kador, P.F. (2010) Ocular Disease: Mechanisms and Management. Saunders/Elsevier, Washington DC, Chapter 32, 243-249.

[16] Lee, A.Y. and Chung, S.S. (1999) Contributions of Polyol Pathway Oxidative Stress in Diabetic Cataract. The FASEB Journal, 13, 23-30. https://doi.org/10.1096/fasebj.13.1.23

[17] Rolo, A.P. and Palmeira, C.M. (2006) Diabetes and Mitochondrial Function: Role of Hyperglycemia and Oxidativestress. Toxicology and Applied Pharmacology, 212, 167-178.

[18] Mulhern, M.L., Madson, C.J., Danford, A., et al. (2006) Theunfolded Protein Response in Lens Epithelial Cells from Galactosemic Rat Lenses. Investigative Ophthalmology \& Visual Science, 47, 3951-3959.

[19] Peppa, M., Uribarri, J. and Vlassara, H. (2003) Glucose, Advanced Glycation End Products, and Diabetes Complications: What Is New and What Works. Clinical Diabetes, 21, 186-187. https://doi.org/10.2337/diaclin.21.4.186

[20] Bron, A.J., Brown, N.A., Harding, J.J., et al. (1998) The Lens and Cataract in Diabetes. International Ophthalmology Clinics, 38, 37-67. https://doi.org/10.1097/00004397-199803820-00005

[21] Creighton, M.O., Trevithick, J.R., Mousa, G.Y., et al. (1978) Globular Bodies: A Primary Cause of the Opacity in Senile and Diabetic Posterior Cortical Subcapsular Cataracts? Canadian Journal of Ophthalmology, 13, 166-181.

[22] Struck, H.G., Heider, C. and Lautenschlager, C. (2000) Changes in the Lens Epithelium of Diabetic and Non-Diabetic Patients with Various Forms of Opacities in Senile Cataract. Klinische Monatsblätter für Augenheilkunde, 216, 204-209.

[23] Straatsma, B.R., Lightfoot, D.O., Barke, R.M. and Horwitz, J. (1991) Lens Capsule and Epithelium in Age-Related Cataract. American Journal of Ophthalmology, 112, 283-296. https://doi.org/10.1016/S0002-9394(14)76729-7

[24] Mulhern, M.L., Madson, C.J., Danford, A., Ikesugi, K., Kador, P.F. and Shinohara, T. (2006) The Unfolded Protein Response in Lens Epithelial Cells from Galactosemic Rat Lenses. Investigative Ophthalmology \& Visual Science, 47, 3951-3959. https://doi.org/10.1167/iovs.06-0193

[25] Brantley, M.A., Umfress, A.C. and Sternberg, P. (2017) Mechanisms of Oxidative Stress in Retinal Injury. In: Schachat, A.P., Sadda, S.R, Hinton, D.R. and Wiedemann, P., Eds., Ryan's Retina, 6th Edition, Elsevier, New York, 582-595. 